The Astrophysical Journal, Vol. 155, January 1969

\title{
THE LOCALLY ISOTROPIC SOLUTIONS OF THE LIOUVILLE AND POISSON EQUATIONS
}

\author{
JÜrgen Ehlers ANd William Rienstra \\ University of Texas at Austin \\ Received April 22, 1968
}

\begin{abstract}
The general solution $(\phi, f)$ of the Liouville equation for a collisionless system of gravitationally interacting particles in which the distribution function $f$ is locally isotropic in momentum space is derived. The distribution function is shown to be a spherical distribution in Chandrasekhar's sense. In the nonstationary case, Poisson's equation is solved and is seen to imply a spatially constant density. The stellar orbits are determined, and a class of anisotropic distribution functions which are also compatible with the previously established potential $\phi$ is constructed. The relation of these solutions to Newtonian cosmology is pointed out.
\end{abstract}

\section{INTRODUCTION}

This paper is devoted to the study of a particularly simple class of solutions of the equations of (Newtonian) stellar dynamics for collision-free systems of gravitating particles.

In $\S$ II we state the basic assumptions of Newtonian stellar dynamics, emphasizing the invariance of this theory not only under Galilean transformations but also under the much larger group of "Newtonian transformations" (eq. [2]), whose importance for spatially unbounded matter distributions has been pointed out by Heckmann and Schücking (1955, 1956, 1959; see also Trautman 1964).

In § III we derive the general solution $(\phi, f)$ of the Liouville equation (6) in which the distribution of residual velocities is everywhere and at all times invariant under all rotations. The a priori restriction (eq. [18]) which we impose on $f$ is much weaker than that for a spherical distribution in the sense of Chandrasekhar (1960, eq. [4.101]). We prove from Liouville's equation that, in fact, every locally isotropic distribution must be of the Chandrasekhar type. At the same time, our proof of this theorem constitutes a simplified rederivation of Chandrasekhar's results on spherical distributions; the resulting solution $(\phi, f)$ is also slightly simpler than Chandrasekhar's because of our use of "Newtonian invariance" (It turns out that Chandrasekhar's arbitrary function $\delta(t)$ can be put equal to zero without loss of generality; this clarifies the origin of the spiral curves discussed in Chandrasekhar 1960, pp. 150 ff.)

In $\S$ IV we determine the general solution $(\phi, f)$ of the coupled Liouville and Poisson equations with locally isotropic distributions $f$. There are two classes of such solutions. The first consists of stationary solutions, i.e., those which are time-independent in a suitable, (possibly) rigidly rotating reference frame; their distributions depend on the energy (relative to the rotating frame) only. The second class consists of time-dependent solutions. They are spatially homogeneous and axially symmetric; their mean motion is a superposition of an isotropic expansion and a rigid rotation. These solutions generalize a class of non-rotating, expanding solutions attributed to Boltzmann (see Kurth 1957, pp. 155-156). These non-stationary solutions can be considered a stellar-dynamical version of those homogeneous Newtonian cosmological models in which the substratum moves shear-free (Heckmann and Schücking 1955, § III).

In $\S \mathrm{V}$ the orbits of particles in the potentials of $\S I V$ are derived. The equations of motion admit four independent first integrals according to the symmetry group of the potential; these integrals are given. With their help, a class of anisotropic distributions 
is constructed in § VI which obey the Liouville equation with the potential from § IV, and which have the same star density as the isotropic distributions derived previously. Hence, a set $(\phi, f)$ of solutions of the coupled Liouville and Poisson equations is obtained in which the distribution of residual velocities is asymmetric, whereas the potential and density are identical with those of the isotropic models. These latter solutions generalize some results of Mjolsness (1968). They exhibit a number of properties which are qualitatively similar to those of certain approximate models of the local cluster of which the Sun is a member (see Ogorodnikov 1965, chap. vii), as pointed out at the end of $\S$ VI.

The general-relativistic analogues of theorems 1 and 2 of this paper have been derived by Ehlers, Geren, and Sachs (1968). In contrast to the Newtonian situation, there the local isotropy of the distribution function excludes, at least for particles of positive rest mass, the possibility of rotation. This difference between Newtonian and Einsteinian stellar dynamics is essentially due to the different underlying kinematics, and does not depend on Einstein's field equation.

\section{BASIC ASSUMPTIONS AND DEFINITIONS}

Since we wish to consider spatially unbounded systems of gravitationally interacting particles, we first recall the assumptions on which the "Newtonian" theory of infinite mass distributions is based (see Heckmann and Schücking 1955, 1956, 1959; see also Trautman 1964).

It is postulated that there exist frames of reference $\left(x^{\prime}, t\right)$ such that freely falling particles, i.e., particles subject only to inertial and gravitational forces, obey

$$
\frac{d^{2} x}{d t^{2}}=-\nabla \phi(x, t)
$$

$\phi(x, t)$ is called the gravitational potential relative to the frame.

If $(x, t)$ is one such "Newtonian" frame, ${ }^{1}$ then $\left(x^{\prime}, t\right)$ is another one if and only if the new spatial axes have constant directions with respect to the old ones; we then write

$$
x^{\prime}=x-d(t),
$$

where $d(t)$ is arbitrary and describes the translational motion of the new frame relative to the old one.

The gravitational potential $\phi$ is not a scalar with respect to the transformations (2); form-invariance of equation (1) requires that

$$
\phi^{\prime}\left(x^{\prime}, t\right)=\phi(x, t)+x \cdot \frac{d^{2} d}{d t^{2}}+h(t) .
$$

The function $h(t)$ is arbitrary; $\phi$ is determined (in any frame) up to an additive function of the time only.

Let us now consider a collection of (possibly infinitely) many particles, each having mass $m$. We describe it statistically in terms of the one-particle distribution function (frequency function) $f(\boldsymbol{x}, t, \boldsymbol{p})$, which measures the density of particle states in $(\boldsymbol{x}, \boldsymbol{p})$ phase space at time $t, p$ being the momentum of a particle.

The mean mass density is

$$
\rho(x, t)=m \int f(x, t, p) d^{3} p,
$$

where the integration is over the whole $p$-space and $f$ is assumed to decrease for large momenta such that the integral converges.

\footnotetext{
1 We avoid the name "inertial frame" or "Galilean frame," since the corresponding concepts are meaningful only for bounded matter distributions; see Heckmann and Schücking 1955, 1956, 1959; see also Trautman 1964.
} 


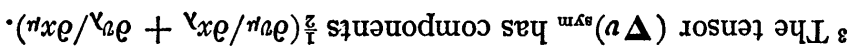

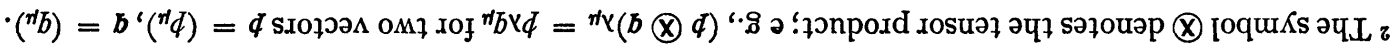

$$
\cdot \frac{t p}{a G}=\boldsymbol{D}
$$

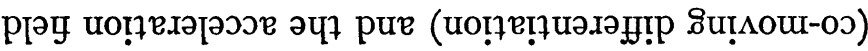

$$
\Delta \cdot \Omega+\frac{\imath e}{e}=\frac{t p}{a}
$$

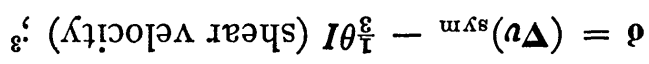

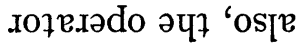

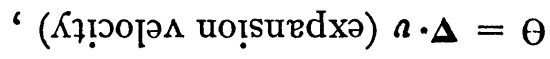

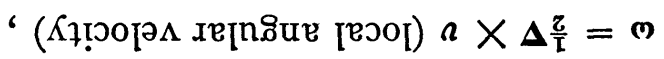

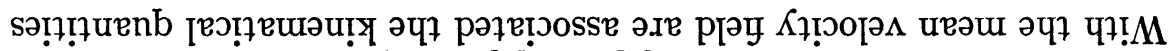

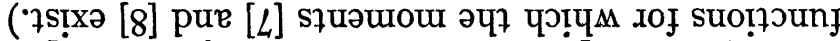

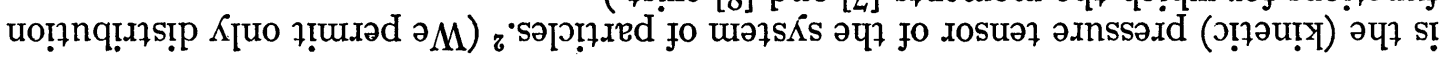

$$
\phi_{\varepsilon} p f(a m-\phi) \otimes(a m-\phi) \int \frac{m}{I}=\left(l^{b} x\right) d
$$

pue '

$$
\phi_{\varepsilon} p\left(\phi^{6} \gamma^{6} x\right) f \phi \int \frac{d}{L}=\left(7^{6} x\right) a
$$

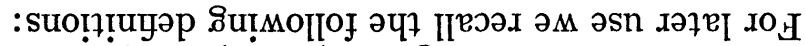

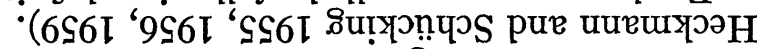

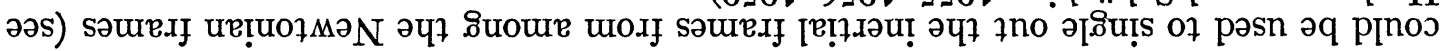

$$
0=\left(f^{6} x\right) \phi \stackrel{\infty}{\leftarrow !} \leftarrow^{x}
$$

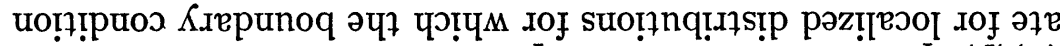

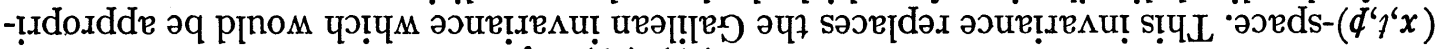

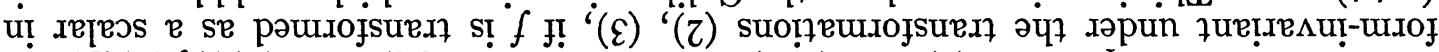

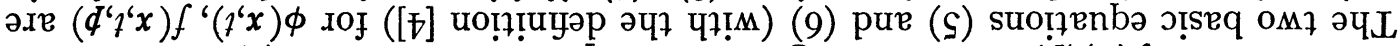

(I) uọ̣ou jo suọ̣?

$$
{ }^{\prime} 0=\frac{\phi e}{f e} \cdot \phi \Delta u-\frac{x e}{f e} \cdot \frac{u}{\phi}+\frac{1 e}{f e}
$$

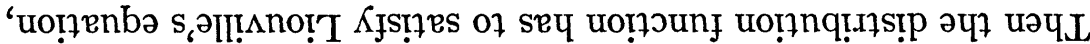

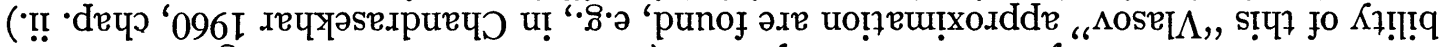

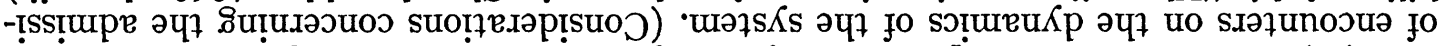

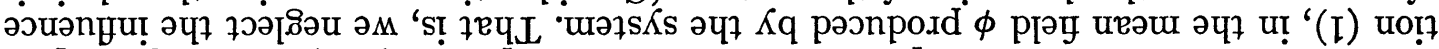
- ६nbə o7 .

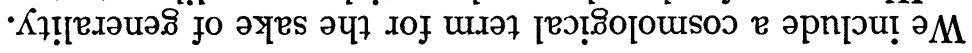

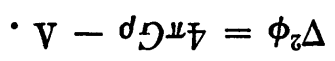

'uo!̣

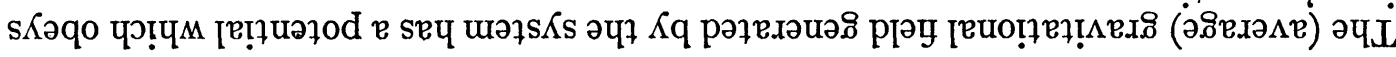


Concerning these quantities, we state a simple lemma which we shall use later.

Lemma (Trümper 1967). - If a velocity field $v(x, t)$ is shear-free, $\boldsymbol{\sigma}=0$, and if its acceleration field is irrotational, $\nabla \times a=0$, then there exists a Newtonian frame $(x, t)$, an expansion function $R(t)>0$, and a (constant) vector $\Omega$ such that (with respect to the mentioned frame)

$$
\begin{gathered}
v=\frac{1}{R} \frac{d R}{d t} x+\frac{\Omega}{R^{2}} \times x, \\
\theta=3 \frac{1}{R} \frac{d R}{d t}, \\
a=\frac{1}{R} \frac{d^{2} R}{d t^{2}} x+\frac{\Omega \times(\boldsymbol{\Omega} \times x)}{R^{4}}=\nabla\left(\frac{1}{2 R} \frac{d^{2} R}{d t^{2}} x^{2}-\frac{(\Omega \times x)^{2}}{2 R^{4}}\right), \\
\nabla \cdot a=\frac{3}{R} \frac{d^{2} R}{d t^{2}}-\frac{2 \Omega^{2}}{R^{4}}, \quad \omega=\frac{\Omega}{R^{2}} ;
\end{gathered}
$$

conversely, equation (14) implies $\boldsymbol{\delta}=0$ and $\boldsymbol{\nabla} \times \boldsymbol{a}=0$.

The flow described in the lemma is the superposition of an isotropic expansion and a spatially constant, time-varying rotation; the distances between any two elements participating in the motion vary as $R(t)$.

III. THE LOCALLY ISOTROPIC SOLUTIONS OF LIOUVILLE'S EQUATION

Let us call a distribution locally isotropic if it has the form

$$
f(x, t, p)=g\left(x, t,[p-m v(x, t)]^{2}\right),
$$

where $g$ is some function of five real variables and $v(x, t)$ is some velocity field. (If $f$ has the form of eq. [18], $v$ necessarily coincides with the mean velocity defined in eq. [7].) These frequency functions $f$ can be characterized by the property that the distribution of peculiar velocities $(p / m)-v(x, t)$ is invariant under all rotations at all places and times $(x, t)$.

Our aim in this section is to find the most general solution $(\phi, f)$ of the Liouville equation (6) with isotropic distribution $f$; we disregard the Poisson equation (5) until the next section. The problem can also be formulated thus: ${ }^{4}$ Find the most general potential $\phi(x, t)$ which admits a first integral $f$ of the locally isotropic form (eq. [18]), and determine, for those potentials, the functions $g$.

To solve this problem, we insert expression (18) into the Liouville equation (6). Using the independent variables $\left(x, t, \eta^{2}, e\right)$ instead of $(x, t, p)$, where

and writing

$$
p-m v=\eta e, \quad e^{2}=1,
$$

$$
g^{\prime}=\frac{\partial g}{\partial\left(\eta^{2}\right)}
$$

we obtain (with definitions [10], [11], [12], and [13]):

$$
\frac{D g}{d t}-\frac{2}{3} \theta \eta^{2} g^{\prime}+\eta e \cdot\left[\frac{1}{m} \nabla g-2 m g^{\prime}(\nabla \phi+a)\right]-2 \eta^{2} g^{\prime}(e \cdot \boldsymbol{\sigma} \cdot e)=0 .
$$

Here and in the sequel, the operations $D / d t$ and $\nabla$ are to be applied to $g\left(x, t, \eta^{2}\right)$ for constant $\eta^{2}$. Equations like (21) hold, according to their derivation, for all values of $x$, $t, \eta^{2}$, and unit vectors $e$.

${ }^{4}$ Compare Chandrasekhar 1960, p. 90, for the formulation of a similar problem. 
For fixed $x, t, \eta^{2}$, the left-hand side of equation (21) is a sum of spherical harmonics of degrees 0,1 , and 2 on the sphere $e^{2}=1$; hence equation (21) is equivalent to the set of three equations ${ }^{5}$

$$
\begin{aligned}
\boldsymbol{\sigma} & =0, \\
\nabla g & =2 m^{2} g^{\prime}(\nabla \phi+a), \\
\frac{D g}{d t} & =\frac{2}{3} \theta \eta^{2} g^{\prime} .
\end{aligned}
$$

Differentiation of equation (23) with respect to $\eta^{2}$ gives $\nabla g^{\prime} \propto \nabla \phi+a$; hence application of the curl operator to equation (23) results in 6

$$
\nabla \times a=0 \text {. }
$$

Equations (25) and (22) show that Trümper's lemma can be applied, i.e., the mean flow of an isotropic distribution satisfying the Liouville equation has the form of equation (14), so that we can use formulae (15), (16), and (17) in the sequel.

If we insert equations (16) and (15) into equations (23) and (24), respectively, we get

$$
\nabla g=g^{\prime} \nabla U, \quad U=2 m^{2}\left[\phi+\frac{1}{2 R} \frac{d^{2} R}{d t^{2}} x^{2}-\frac{(\Omega \times x)^{2}}{2 R^{4}}\right],
$$

and

$$
R^{2} \frac{D g}{d t}=\eta^{2} g^{\prime} \frac{d\left(R^{2}\right)}{d t}
$$

Equation (26) implies that, for fixed $t$ and $\eta^{2}, g$ and $U$ are functionally dependent. Hence there exists a function $F$ of three variables such that

$$
g=F\left(R^{2} U, R^{2} \eta^{2}, t\right),
$$

identically in $x$, $t$, and $\eta^{2}$. (It is convenient to use $R^{2} U$ and $R^{2} \eta^{2}$ rather than $U$ and $\eta^{2}$ as arguments of $F$, as will be seen.)

With equation (28), equations (26) and (27) become

$$
\begin{gathered}
{\left[\frac{\partial F}{\partial\left(R^{2} U\right)}-\frac{\partial F}{\partial\left(R^{2} \eta^{2}\right)}\right] \nabla U=0} \\
-\frac{\partial F}{\partial t}=\frac{\partial F}{\partial\left(R^{2} U\right)} \frac{D\left(R^{2} U\right)}{d t} .
\end{gathered}
$$

If $\nabla U \neq 0$, equation (29) implies that $F$ depends on $t$ and $R^{2} U+R^{2} \eta^{2}$ only,

$$
g=H\left(R^{2}\left[U+\eta^{2}\right], t\right) \text {. }
$$

For no value of $t$ can the function $H$ be constant with respect to its first argument, since it represents a distribution function (see the argument in $\mathrm{n} .5$ above). Consequently, for some values of $\eta^{2}$ it is permissible to rewrite equation (30) as

$$
-\frac{\partial H}{\partial t} / \frac{\partial H}{\partial(. .)}=\frac{D\left(R^{2} U\right)}{d t} \text {. }
$$

- At any event $(x, t), g^{\prime} \neq 0$ for some $\eta^{2}$, since $g>0$ and

$$
\lim _{\eta \rightarrow \infty} g=0 \text {. }
$$

This is used in obtaining equation (22) and, later, equation (25).

'See n. 5 above. 
Since the right-hand side depends on $x$ and $t$, not on $\eta^{2}$, the function on the left must be constant with respect to its first argument, $R^{2}\left[U+\eta^{2}\right]$. Hence, both sides depend on $t$ only. However, equation (26) shows that a transformation $\phi \rightarrow \phi+h(t)$ changes $D\left(R^{2} U\right) / d t$ by an arbitrary, additive function of $t$. Consequently, without loss of generality we may require that

$$
\frac{D\left(R^{2} U\right)}{d t}=0
$$

Equations (30) and (31) then show that

$$
g=H\left(R^{2}\left[U+\eta^{2}\right]\right) \text {. }
$$

It is easy to prove that, if $\nabla U=0$, the last two formulae again hold, and that without loss of generality we can put $U=0$.

The general solution of equation (33) for a flow of the form of equation (14) is given, in terms of an arbitrary function $\psi$ of a vector argument, by

where

$$
R^{2} U=\psi\left(e^{\Omega^{*} \tau} \cdot \frac{x}{R}\right)
$$

$$
\tau=\int_{t_{0}}^{t} \frac{d t^{\prime}}{R^{2}\left(t^{\prime}\right)}
$$

Here the skew tensor $\mathbf{\Omega}^{*}$ is dual to the vector $\boldsymbol{\Omega}$, i.e.,

$$
\mathbf{\Omega}^{*}=\left|\begin{array}{rrr}
0 & \Omega_{3} & -\Omega_{2} \\
-\Omega_{3} & 0 & \Omega_{1} \\
\Omega_{2} & -\Omega_{1} & 0
\end{array}\right|, \quad \mathbf{\Omega}=\left(\Omega_{1}, \Omega_{2}, \Omega_{3}\right) .
$$

We note in passing a result, needed in the next section, which follows by straightforward calculation from equation (35):

$$
\frac{D}{d t}\left(R^{4} \nabla^{2} U\right)=0 .
$$

We summarize the main results of this section in theorem 1. The most general potential for which the equation of motion (1) possesses a first integral of the form $g\left(x, t, m^{2}[\dot{x}-v(x, t)]^{2}\right)$ (with some mean velocity field $v$ which is not specified a priori) is given $b y^{7}$

$$
\phi=\frac{(\Omega \times x)^{2}}{2 R^{4}}-\frac{1}{2 R} \frac{d^{2} R}{d t^{2}} x^{2}+\left(2 m^{2} R^{2}\right)^{-1} \psi\left(e^{\Omega^{*} \tau} \cdot \frac{x}{R}\right)
$$

where $t_{0}$ is a constant (initial) time, $\mathbf{\Omega}^{*}$ a constant skew tensor, $R(t)$ an arbitrary positive function of the time, and $\psi(y)$ an arbitrary real function of a vector argument, and where $\tau(t)$ is defined by equation (36). The corresponding mean velocity field is that given by equation (14), and the first integral is of the form

$$
H\left(R^{2}\left[U+m^{2}\{\dot{x}-v\}^{2}\right]\right),
$$

${ }^{7}$ In a suitable Newtonian frame, i.e., modulo transformations (2), (3). 
where $U$ is defined by equation (35) and $H$ is an arbitrary real function of one real variable. If $H$ is chosen non-negative and decreasing at infinity such that

$$
\int_{0}^{\infty} H(u) u^{2} d u<\infty
$$

then the pair $(\phi, f)$ with $f=H$ represents the most general solution of the Liouville equation (6) with a locally isotropic distribution $f$. In the latter case, the pressure is isotropic, $\boldsymbol{P}=p I$, and density and pressure are given by

$$
\begin{aligned}
& \rho=\frac{4 \pi m}{R^{3}} \int_{0}^{\infty} H\left(R^{2} U+\xi^{2}\right) \xi^{2} d \xi, \\
& p=\frac{4 \pi}{3 m R^{5}} \int_{0}^{\infty} H\left(R^{2} U+\xi^{2}\right) \xi^{4} d \xi
\end{aligned}
$$

so that

$$
\frac{D\left(\rho R^{3}\right)}{d t}=0
$$

(mass conservation) and

$$
\frac{D}{d t}\left(\frac{p^{3}}{\rho^{5}}\right)=0,
$$

corresponding to a polytropic index $\frac{3}{2}$. At any time, density and pressure depend functionally on $U$.

\section{RESTRICTIONS IMPOSED BY POISSON'S EQUATION}

We now investigate which of the solutions $(\phi, f)$ of Liouville's equation described in theorem 1 also obey the (generalized) Poisson equation (5).

If $R=$ const., the mean flow (eq. [14]) is a stationary, rigid rotation with angular velocity $\omega=R^{-2} \boldsymbol{\Omega}$, and equations (33), (42) show that $U$ and $\rho$ are time-independent in a co-rotating frame of reference. Hence, Poisson's equation will always be satisfied if it holds at one time.

If we write $U$ instead of $U / 2 m^{2}$, insert the second of equations (26) and equation (40) into Poisson's equation (5), change the variable of integration $(\xi \rightarrow m R \xi)$ and write $H(x)$ instead of $H\left(2 R^{2} m^{2} x\right)$, we obtain as the equations governing the stationary solutions of equations (5), (6), and (18):

$$
\begin{aligned}
\nabla^{2} U+2 \omega^{2}+\Lambda & =(4 \pi)^{2} G m^{4} \int_{0}^{\infty} H\left(U+\frac{1}{2} \xi^{2}\right) \xi^{2} d \xi, \\
f(x, t, p) & =H\left(U+\frac{1}{2}\left[\frac{p}{m}-v\right]^{2}\right) \\
\phi & =U+\frac{1}{2}(\omega \times x)^{2} .
\end{aligned}
$$

Special solutions of these equations with $\omega=0, \Lambda=0$, are provided by isothermal and polytropic gas spheres (see, e.g., Chandrasekhar 1957, chap. iv). Another simple case, with $\omega \neq 0$, is obtained by putting $U=0$; this case corresponds to the rigidly rotating, stationary, homogeneous model universes of Heckmann and Schücking (1955).

We do not consider the case $R=$ const. further here, but turn our attention now to 
the case where $R$ is not a constant. Inserting the second of equations (26) into Poisson's equation (5), we obtain

$$
4 \pi G \rho R^{3}=\left(2 m^{2} R\right)^{-1}\left(R^{4} \nabla^{2} U\right)-3 R^{2} \frac{d^{2} R}{d t^{2}}+2 \frac{\Omega^{2}}{R}+\Lambda R^{3} .
$$

Taking into account equations (42) and (38), we differentiate this equation along the streamlines:

$$
\frac{R^{2} \nabla^{2} U}{2 m^{2}} \frac{d R}{d t}=\frac{d}{d t}\left(2 \frac{\Omega^{2}}{R}-3 R^{2} \frac{d^{2} R}{d t^{2}}+\Lambda R^{3}\right) .
$$

Since $d R / d t \neq 0$ (except at special instants), it follows from this relation that $U$ depends on the time only; hence (see eq. [26]) $\nabla^{2} \phi$ depends on $t$ only, so that $\rho=\rho(t)$ and

$$
\frac{8 \pi G}{3} \rho R^{3}=C=\text { const. }
$$

Equation (40) then implies that $R^{2} U=$ const., for the integral is a strictly monotonic function of $R^{2} U$ :

$$
\begin{aligned}
\frac{d}{a\left(R^{2} U\right)} \int_{0}^{\infty} H\left(R^{2} U+\xi^{2}\right) \xi^{2} d \xi & =\int_{0}^{\infty} H^{\prime}\left(R^{2} U+\xi^{2}\right) \xi^{2} d \xi \\
=\frac{1}{2} & \int_{0}^{\infty}\left[\frac{\partial}{\partial \xi} H\left(R^{2} U+\xi^{2}\right)\right] \xi d \xi=-\frac{1}{2} \int_{0}^{\infty} H\left(R^{2} U+\xi^{2}\right) d \xi<0 .
\end{aligned}
$$

The last term in equation (39), being a constant, can therefore be omitted from the potential $\phi$, and the notational change $H\left(R^{2} U+\xi^{2}\right) \rightarrow H\left(\xi^{2}\right)$ then eliminates $U$ completely from the basic equations of theorem 1 .

Poisson's equation (47) reduces to the differential equation

$$
\frac{d^{2} R}{d t^{2}}+\frac{C}{2 R^{2}}-\frac{2}{3} \frac{\mathbf{\Omega}^{2}}{R^{3}}-\frac{\Lambda}{3} R=0
$$

for the expansion function $R(t)$; it can be integrated once to yield

$$
\left(\frac{d R}{d t}\right)^{2}-\frac{C}{R}+\frac{2}{3} \frac{\Omega^{2}}{R^{2}}-\frac{\Lambda}{3} R^{2}=-k c^{2}=\text { const. }
$$

The (dimensionless) constant of integration $k$ can be chosen to be $+1,-1$, or 0 . By this convention the scale of $R$ is uniquely determined if $k \neq 0$. We have thus established the theorem 2. The most general solution $(\phi, f)$ of the Poisson and Liouville equations (5), (6) with a locally isotropic distribution function $f$ (see eq. [18]) and a non-stationary mean velocity $v$ is given by ${ }^{8}$

$$
\begin{gathered}
\phi=\left(\frac{C}{4 R^{3}}+\frac{\boldsymbol{\Omega}^{2}}{6 R^{4}}-\frac{\Lambda}{6}\right) x^{2}-\frac{(\Omega \cdot x)^{2}}{2 R^{4}}=-\frac{1}{2 R} \frac{d^{2} R}{d t^{2}} x^{2}+\frac{1}{2 R^{4}}(\Omega \times x)^{2}, \\
f=H\left(R^{2}[p-m v]^{2}\right), \\
v=x \sqrt{ }\left(\frac{1}{3} \Lambda+\frac{C}{R^{3}}-\frac{2 \Omega^{2}}{3 R^{4}}-\frac{k c^{2}}{R^{2}}\right)+\frac{\Omega \times x}{R^{2}}, \\
\rho=\frac{3 C}{8 \pi G R^{3}},
\end{gathered}
$$

${ }^{8}$ See $n .7$ above. 


$$
p=\frac{4}{3 m R^{5}} \int_{0}^{\infty} H\left(\xi^{2}\right) \xi^{4} d \xi
$$

Here, $\boldsymbol{\Omega}$ is an arbitrary constant vector; $k=1,-1$, or 0 ; and $H$ is a non-negative function such that

$$
C=\frac{\mathbf{3 2}}{\mathbf{3}} \pi^{2} G m \int_{0}^{\infty} H\left(\xi^{2}\right) \xi^{2} d \xi
$$

exists and is positive. The expansion $R(t)$ is a non-constant, positive solution of equation (51).

Equations (50) and (51) are well known in Newtonian cosmology; they govern the expansion of the (spatially) homogeneous cosmological models in which the substratum moves shear-free (see Heckmann and Schücking 1955, §3). In fact, equations (49), (52), and (54) are identical with those which describe the density, mean velocity, and potential of those models. Theorem 2 can, therefore, be regarded as a new characterization of these models, with the modification that here the matter is treated, not as a continuous medium, but as an ensemble of particles. This last circumstance implies that the pressure, which remains completely arbitrary in the hydrodynamical treatment, is now determined by the distribution of residual velocities. The homogeneity of the solutions described in theorem 2 follows from the fact that the functions $(\phi, f)$ (and, consequently, $v, \rho, p)$, if properly transformed, are invariant under the three-parameter group of transformations,

$$
x^{\prime}=x-R e^{-\Omega^{*} r} \cdot \beta \quad(\beta=\text { const. }),
$$

from one "fundamental observer" to another. (The function $\tau(t)$ is defined, as before, by eq. [36].)

The axial symmetry of these models follows from the invariance under the rotations

$$
x^{\prime}=e^{\Omega^{* a}} \cdot \boldsymbol{x} \quad(a=\text { const. })
$$

about the axis $\boldsymbol{\Omega}$. As a consequence of theorem 2, the cosmological principle (see, e.g., Bondi 1960) can be replaced, in the stellar-dynamical approach presented here, by the postulates that (a) the distribution of residual velocities is everywhere and at all times isotropic with respect to the fundamental observers, and (b) (to insure macroscopic isotropy) the mean flow is irrotational.

An elegant qualitative discussion of the variety of solutions of equations (50) and (51) has been given by Heckmann and Schücking (1955, §3). The most important consequence of rotation is the absence of a density singularity; the centrifugal potential barrier forces $R$ to have a positive minimum. Hence, truly periodic or oscillating model universes are possible. (To call non-rotating $k=1$ models "oscillating" is misleading, since the corresponding solutions are regular only in open time intervals between successive zeros of $R(t)$.)

In the case $\Lambda=0$, equation (51) can be solved by elementary functions:

$$
\begin{aligned}
& k=1: c^{3} t=\frac{1}{4} \pi C+\frac{1}{2} C \operatorname{arc} \sin \left[\frac{1}{a}\left(\frac{2 c^{2} R}{C}-1\right)\right]-c \sqrt{ }\left(C R-\frac{2}{3} \Omega^{2}-c^{2} R^{2}\right), \\
& k=0: C t=\frac{2}{3}\left(R+\frac{4}{3} \frac{\boldsymbol{\Omega}^{2}}{C} \sqrt{ }\left(R-\frac{2}{3} \frac{\Omega^{2}}{C}\right),\right. \\
& k=-1: c^{3} t=-\frac{1}{2} C \operatorname{arc} \cosh \left[\frac{1}{a}\left(\frac{2 c^{2} R}{C}+1\right)\right]+c \sqrt{ }\left(C R-\frac{2}{3} \boldsymbol{\Omega}^{2}+c^{2} R^{2}\right),
\end{aligned}
$$


where

$$
a=\sqrt{ }\left(1+k \frac{8}{3} c^{2} \frac{\boldsymbol{\Omega}^{2}}{C^{2}}\right) .
$$

The oscillating model, $k=1$, has a period of

$$
T=\pi \frac{C}{c^{3}}=\frac{8 \pi^{2}}{3 c^{3}} G \rho R^{3} .
$$

In the cases $k=-1$ and $k=0$, the "universe" contracts from an infinitely dispersed state, reaches a state of maximum density and angular velocity, and then expands indefinitely.

Since the density of the non-stationary models considered here is spatially constant, these solutions are useless as models for localized systems like galaxies or clusters, except, perhaps, if one restricts their application to the central regions of some systems like globular clusters. Stationary systems, as given by equations (44), (45), and (46), may have spatially varying mass distributions, which are carried around rigidly. Thus, the proposal of Chandrasekhar (1960, chap. iv) to use models with a spherical distribution of residual velocities to describe spiral patterns is feasible, at best, in the stationary case.

\section{PARTICLE ORBITS}

The potential $\phi$ given by equation (52) can be obtained as follows. Consider the Lagrangian

$$
L^{*}=\frac{1}{2}\left(\frac{d \xi}{d \tau}\right)^{2}-\frac{1}{2}(\Omega \times \xi)^{2} \quad(\Omega=\text { const. })
$$

of an anisotropic harmonic oscillator, and introduce new space and time coordinates $(x, t)$ by means of the transformation (cf. Schürer 1943; Kurth 1957, pp. 154-155)

$$
x=R(t) \xi, \quad \tau=\int_{t_{0}}^{t} \frac{d t^{\prime}}{R^{2}\left(t^{\prime}\right)} .
$$

The Lagrangian $L$ corresponding to the new variables is, from Hamilton's principle, $L=L^{*} d \tau / d t=L^{*} R^{-2}$, and is found to be

$$
L=\frac{1}{2}\left(\frac{d x}{d t}\right)^{2}-\phi
$$

with $\phi$ given by equation (52).

Since the general orbit corresponding to $L^{*}$ is the superposition of a uniform motion in the $\mathbf{\Omega}$-direction and an elliptic oscillation with circular frequency $|\boldsymbol{\Omega}|$ in the plane orthogonal to $\mathbf{\Omega}$, the general orbit corresponding to $L$ is immediately obtained from transformation (63). In rectangular Cartesian coordinates with $\boldsymbol{\Omega}=(0,0, \Omega)$ the general trajectory is given by

$$
x=X R(t) \sin (\Omega \tau+a), \quad y=Y R(t) \sin (\Omega \tau+\beta), \quad z=R(t)(Z+\gamma \tau) .
$$

The quantities $X, Y, Z, a, \beta$, and $\gamma$ are constants, and $\tau$ is given by equation (63).

The Lagrangian $L$ is "essentially invariant" under transformations (57) and (58); i.e., $L\left(x^{\prime}, \dot{x}^{\prime}, t\right)-L(x, \dot{x}, t)$ is a total time derivative. Hence, according to Noether's theorem (see Hill 1951; Trautman 1967), the equations of motion corresponding to $L$ admit four independent first integrals. It is easily checked that the following functions are independent first integrals:

$$
K=R e^{-\Omega^{*} \tau} \cdot(\dot{x}-v)
$$




$$
J=(x \times \dot{x}) \cdot \Omega .
$$

In equation (66), $R$ is the expansion function and $v$ is the mean velocity of theorem 2.

The integral (66) results from the spatial homogeneity of the potential $\phi$, and the angular-momentum integral (67) results from the axial symmetry.

Equation (66) shows that the magnitude of the residual velocity of a particle changes as $R^{-1}$. This fact, well known for the non-rotating models, explains the $R^{-5}$-dependence of the pressure in equation (55). For a Maxwellian distribution it implies a "temperature" $T \propto R^{-2}$.

\section{ANISOTROPIC DISTRIBUTIONS}

According to the preceding section, every function of the four integrals $K, J$ satisfies Liouville's equation (6) for the potential $\phi$ given by equation (52).

Since $\boldsymbol{K} \propto R$, equation (49) shows that distributions of the form

$$
f=H(\boldsymbol{K})=H\left(R e^{-\Omega^{*} \cdot} \cdot\left[\frac{p}{m}-v\right]\right)
$$

have the same density $\rho$ as the isotropic distributions (53) provided $H$ is normalized according to

$$
C=\frac{8 \pi G}{3} m^{4} \mathcal{S} H(\boldsymbol{K}) d^{3} K
$$

The distribution (68) determines a mean velocity

and a pressure tensor

$$
\langle\boldsymbol{v}\rangle=\boldsymbol{v}+R^{-1} e^{\boldsymbol{\Omega}^{*} \boldsymbol{r}} \cdot \boldsymbol{B}, \quad \boldsymbol{B}=\frac{\mathcal{S} K H(\boldsymbol{K}) d^{3} K}{\mathcal{S} H(\boldsymbol{K}) d^{3} K},
$$

$$
P=\frac{m^{4}}{R^{5}} e^{\Omega^{*} \tau} \cdot D \cdot e^{-\Omega^{*} \tau}, \quad D=\mathcal{S} \boldsymbol{X} \otimes K(K+B) d^{3} K .
$$

Since $H$ in equation (68) is an arbitrary non-negative function of $K$, the distribution of residual velocities at one instant $t_{0}$ and one point $x_{0}$ can be prescribed arbitrarily; the distribution is then determined everywhere and at all times through equation (68).

Thus, any potential $\phi$ of the form of equation (52) (with an $R(t)$ satisfying eq. [51]) is compatible with all distributions $f$ of the form of equation (68) (with eq. [69] and $v$ according to eq. [54]), in the sense that $(\phi, f)$ satisfy Poisson's and Liouville's equations. In particular, one obtains in this way a class of solutions of Chandrasekhar's problem if $H$ is specialized to a quadratic form in $K$; this form of $H$ seems, however, in no natural way to be distinguished in the general class of solutions.

According to equation (71), the principal pressures change in time as $R^{-5}$, and the principal axes of the pressure ellipsoid precess with angular velocity $-\boldsymbol{\omega}=R^{-2} \boldsymbol{\Omega}$. In the special case of an ellipsoidal velocity distribution, equation (68) shows that the velocity ellipsoid precesses in the same manner.

This research was supported by Aerospace Research Laboratories, OAR, through grant AF-33(615)1029.

\section{REFERENCES}

Bondi, H. 1960, Cosmology (Cambridge: Cambridge University Press). Chandrasekhar, S. 1957, An Introduction to the Study of Stellar Structure (New York: Dover Publications). - 1960, Principles of Stellar Dynamics (New York: Dover Publications).

Ehlers, J., Geren, P , and Sachs, R. K. 1968, J. Math. Phys., 9, 1344.

Heckmann, O., and Schücking, E 1955, Zs. f. Ap., 38, 95. 
Heckmann, O., and Schücking, E. 1956, ibid., 40, 81.

.1959, "Newtonsche und Einsteinsche Kosmologie," in Hdb.d. Phys., ed. S. Flügge (Berlin: SpringerVerlag), 53, 489.

Hill, E. L. 1951, Rev. Mod. Phys., 23, 253.

Kurth, R. 1957, Introduction to the Mechanics of Stellar Systems (New York: Pergamon Press).

Mjolsness, R. C. 1968, $A$ p.J. (in press).

Ogorodnikov, K. F. 1965, Dynamics of Stellar Systems (New York: Pergamon Press).

Schürer, M. 1943, Astr. Nach., 273, 230.

Trautman, A. 1964, "Foundations and Current Problems of General Relativity," chaps. v and ix in Lectures on General Relativity, Proc. Brandeis Summer Inst. Theoret. Phys, ed. S. Deser and K. W. Ford (London: Prentice-Hall). 1967, Com. Math. Phys., 6, 248.

Trümper, M. 1967, $Z s$ f. $A p, 66,215$.

Copyright 1969. The University of Chicago. Printed in U S A. 